\title{
Graphene-based catalysts for hydrogen evolution reaction
}

\author{
Lana Reagent, Maximina Luis-Sunga, Elena Pastor, Gonzalo García*
}

Instituto Universitario de Materiales y Nanotecnología, Departamento de Química, Universidad de La Laguna, PO Box 456, 38200, La Laguna, Santa Cruz de Tenerife, Spain.

\begin{abstract}
Developing sustainable and renewable energy sources is critical as higher and higher global energy and environmental challenges arise. Hydrogen has the highest mass/energy density of any fuel and is considered one of the best sources of clean energy. Water splitting is regarded as one of the most promising solutions for hydrogen production on a large scale. Highly efficient, durable and cost-effective catalysts for hydrogen evolution reaction (HER) are critical in the realization of this goal. Among many materials proposed, graphene-based materials offer some unique properties for HER catalysis. In this review, we present recent progress on development of graphenebased electrocatalysts toward HER throughout the past few years.
\end{abstract}

Keywords: HER; Graphene; Catalysis; Catalysts; Electrolyser 


\section{Introduction}

Most of the energy produced worldwide comes from fossil fuels, which presents several problems. Firstly, combustion of fossil fuels produces carbon dioxide $\left(\mathrm{CO}_{2}\right)$ which drives on climate change [1]. Secondly, fossil fuels are not a renewable source and once the reserves are exhausted, another form of storing and producing energy will be needed.

In this scenario, water splitting appears to be the best method of converting electrical energy to hydrogen as it requires only water and produces nothing but pure hydrogen $\left(\mathrm{H}_{2}\right)$ and oxygen $\left(\mathrm{O}_{2}\right)$. In a typical water electrolysis system, $\mathrm{H}_{2}$ is produced at the cathode through the hydrogen evolution reaction (HER) and $\mathrm{O}_{2}$ is produced at the anode through the oxygen evolution reaction (OER) [2].

The overall reaction for HER occurs in the whole $\mathrm{pH}$ range in the subsequent way:

$$
\begin{array}{ll}
2 \mathrm{H}^{+}+2 e^{-} \rightarrow \mathrm{H}_{2} & (\mathrm{pH}<7) \\
2 \mathrm{H}_{2} \mathrm{O}+2 e^{-} \rightarrow \mathrm{H}_{2}+2 \mathrm{OH}^{-} & (\mathrm{pH}>7)
\end{array}
$$

Pure hydrogen is a clean energy vector, which can be stored to obtain energy through fuel cell technology. In this way, the energy obtained by renewable energy sources (such as solar, wind, etc.) can be stored in hydrogen form delivering energy without producing any polluting waste when it is required [3]. Hydrogen production by means of water electrolysis is a potential route to an energy sustainable future [4]. Currently, the efficiency of hydrogen production by water electrolysis is too low to be economically competitive for real energy requirements [5]. Consequently, efficient catalysts are sought to ensure good HER performance. In this regard, noble metals, such as platinum, exhibit the highest electrocatalytic activity, but their shortage and high cost restrict their large-scale application [6]. Therefore, efficient and economical catalysts research is crucial for the development of this technology.

\section{Graphene-based materials}

Graphene has attracted extensive attention due to its excellent physical and chemical properties, large surface area, excellent electrical conductivity and high mechanical strength [7]. Pure graphene is catalytically inactive for HER due to its flat and inert surface [8]. Therefore, graphene undergoes different doping, functionalization 
and strain processes with the objective of tune its chemical properties. Fortunately, graphene derivatives such as reduced graphene oxide $(\mathrm{rGO})$ can be synthesized by the simple Hummers method and used as precursor for a wide range of graphene-based materials [9]. Because of residual oxygenated groups and defects, the conductivity of rGO is lower than that of pristine graphene. However, reactive surfaces of GO and rGO enable the optimization of specific properties and ease the incorporation of the dopant or composite.

Doping has been widely investigated in order to specifically tailor the catalytic properties [10]. The creation of charged groups and defects in the graphene network by modifying the carbon atoms surface gives place to defective graphene materials that present an alteration of the geometry and the electronic structure. These materials are used as electrocatalysts since they can induce lower activation barriers or improved adsorption energy to reactants, products or intermediate species, being able to achieve higher catalytic activities or selectivity towards certain reactions [11]. Moreover, it is well known that topological defects or edges are related to the electrocatalytic performance so a large amount of defects can lead to enhance the graphene catalytic activity [12].

\section{Heteroatom-doped metal-free graphene catalysts}

Heteroatom-doped noble metal-free catalysts have been synthesized (i.e., nitrogen, sulphur, fluorine, boron, phosphorus, etc.) tuning its electronic properties for enhancing hydrogen evolution reaction. In this regard, nitrogen doping has been an effective way to tune the properties of graphene contributing to its development for various applications. The nitrogen bonding configuration normally obtained are pyridinic $\mathrm{N}$, pyrrolic $\mathrm{N}$, and graphitic $\mathrm{N}$ [8]. It has also been reported nitrogen and sulphur co-doping leads to higher activity of graphene in HER at low operating potential (Figure 1) obtaining comparable results to $2 \mathrm{D} \mathrm{MoS}_{2}$, the best Pt-free HER catalyst [7]. Also, nitrogen and phosphorus dual-doped graphene has been studied exhibiting comparable onset overpotential, Tafel slope and exchange current density to some of the traditional metallic catalysts $[13,14]$ due to the synergistic effect of the dopants. 

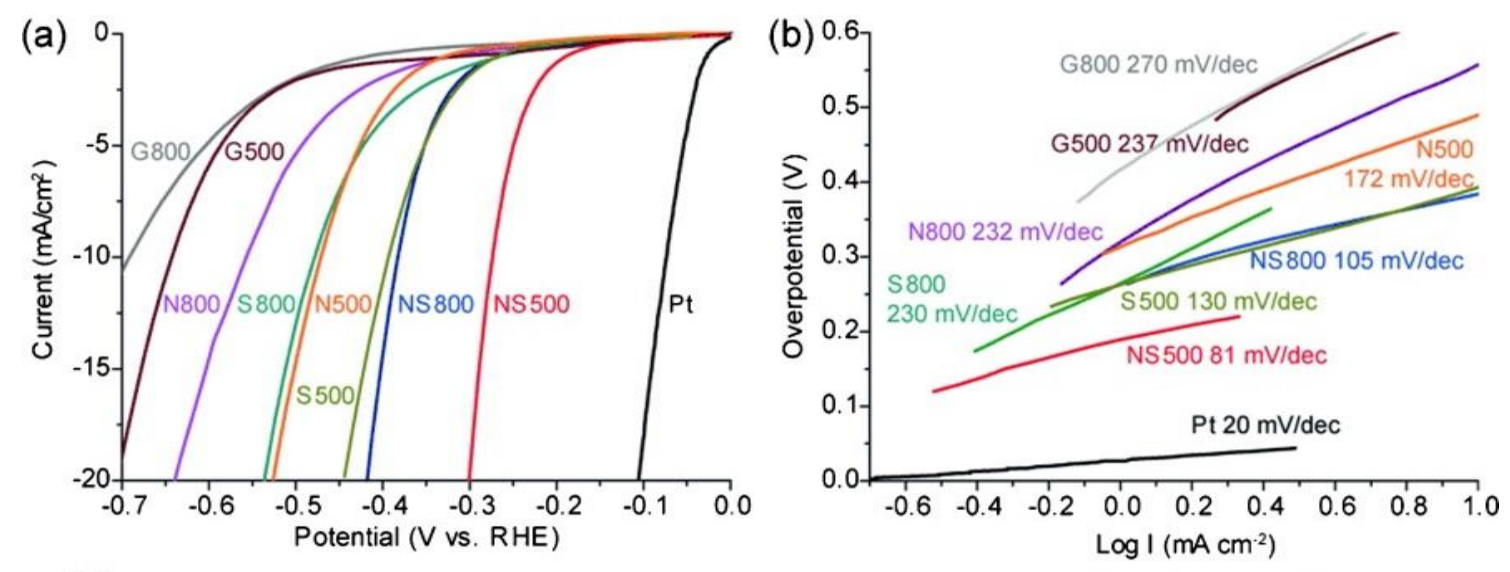

Figure 1. HER activity of chemically doped nanoporous graphenes: G (graphene), N (N-doped graphene), S (S-doped graphene) and NS (NS-doped graphene). a) CV curves of the samples produced at different chemical vapor deposition temperatures and with different dopants in comparison to $\mathrm{Pt}$; b) Tafel plots for the different samples. Reproduced with permission (ref. 7).

Boron has also emerged as doping heteroatom in several technological fields $[11,15]$. It has been demonstrated that B-substituted graphene synthetized by controlled substitution of the $\mathrm{C}$ atoms is an efficient metal-free electrocatalyst for HER [11] and Bdoped graphene can lower the conversion barriers for the transformation of $\mathrm{H}^{+}$ions to $\mathrm{H}_{2}$ showing a better HER activity than undoped graphene [16].

\section{Noble metal-free graphene catalysts}

The nonprecious transition metal insertion ( $\mathrm{Mn}, \mathrm{Co}, \mathrm{Cu}, \mathrm{Ni}$, etc.) has also been investigated obtaining satisfactory results displaying high HER performances due to an active metal-H bond interaction and similar electronic structure to $\mathrm{Pt}[16,17]$. In this field, ultra-small ruthenium phosphide nanoparticles grown on reduced graphene oxide nanosheets were reported has a highly efficient HER catalyst achieving superior current density at extremely low overpotentials than commercial Pt/C [18]. Furthermore, Deng et al. found out increasing the amount of nitrogen doping and reducing the number of graphene layers that encapsulate a CoNi nanoparticle can significantly increase the electron density enhancing the HER activity in acidic media [19]. On the other hand, sulphur-doped graphene has recently attracted as a promising material beyond $\mathrm{N}$-doped graphene displaying to be competitive or even better compared to $\mathrm{N}$-doped materials for ORR activity. Recently it has been investigated as a noble metal-free catalyst for HER in acidic media obtaining a significantly enhance to HER activity of graphene attributed 
to the presence of high S-doping level with thiophene-S rich species [16]. Figure 2 shows the role of S-doping level, species types/contents, and defect level towards HER in acid media.

a

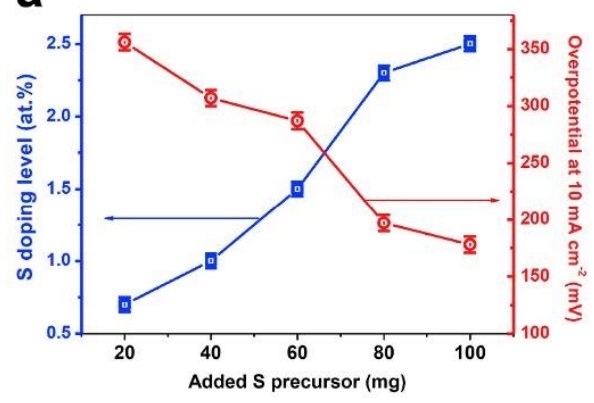

C

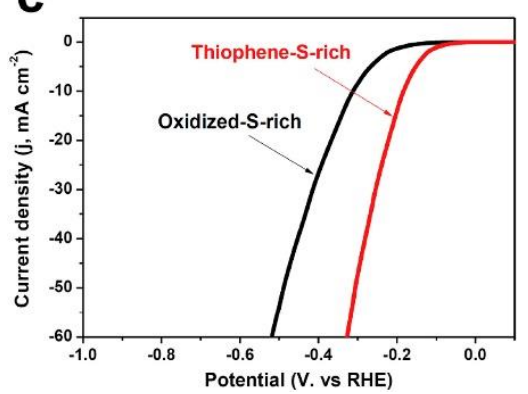

b

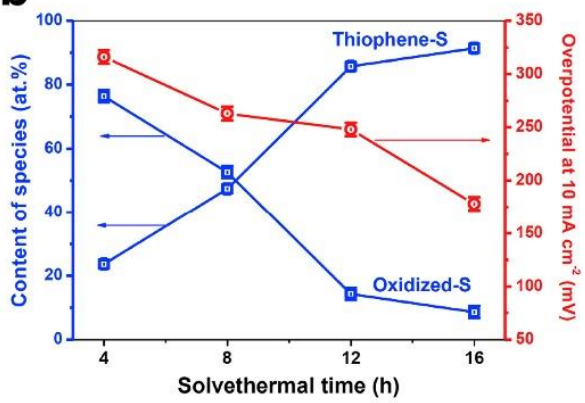

d

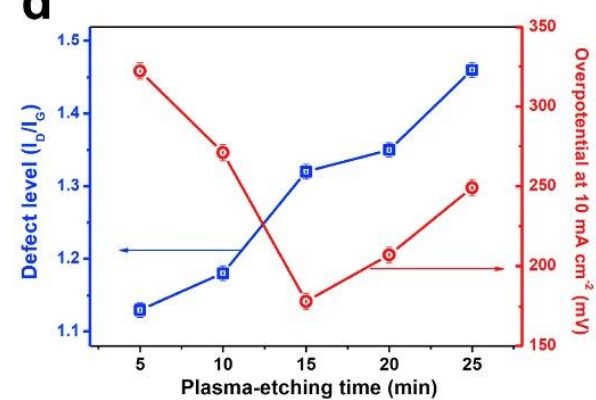

Figure 2. (a) HER activities (overpotential at $10 \mathrm{~mA} \mathrm{~cm}^{-2}$ ) of sulphur-doped graphene (SG) samples with different S-doping levels obtained by varying the amount of added $\mathrm{S}$ precursor. (b) HER activities of SG samples with different compositions of thiopheneS/oxidized-S species obtained by varying the solvothermal time during the synthesis of SG. (c) Polarization curves of thiophene-S-rich and oxidized-S-rich SG samples in $0.5 \mathrm{M} \mathrm{H}_{2} \mathrm{SO}_{4}$ solution at a scan rate of $5 \mathrm{mV} \mathrm{s}^{-1}$. (d) HER activities of SG samples with different topological defect levels $\left(I_{\mathrm{D}} / I_{\mathrm{G}}\right.$ ratio $)$ obtained by varying the plasmaetching time. Reproduced with permission (ref. 16).

In addition, co-doping materials with metal and non-metal pairs with high catalytic performance have also been reported showing dual-doped multilayer graphene exhibit higher HER activity than mono-doped materials as a result of a synergetic dualdoped effect [13]. Regarding this, nitrogen-doped graphene materials are the ones with the highest HER performance [20], especially, $\mathrm{Co}$ and $\mathrm{Cu}$ embedded $\mathrm{N}$-enriched mesoporous carbon showed high catalytic ability in HER [21]. Besides, recent theoretical studies support the HER performance can be dramatically improved on waved graphene due to localized chemical potential and Pt-analogous activity showing $\mathrm{Ni}-\mathrm{N}$ and V-N co-doped graphenes have the highest catalytic ability [22]. Graphene- 
encapsulated CoNi and RuCo nanoalloys have been reported to have a high HER activity due to the thin nitrogen-doped graphene layer protecting the alloy from corrosion and simultaneously promoting the electron penetration of the transition metals to the carbon surface $[19,23]$. A similar synergistic effect has been shown for iron nanoparticles [24].

\section{Graphene-based composite catalysts}

Graphenic materials have been used to synthesize different 1D, 2D and 3D nanocomposites with enhanced catalytic activity toward the HER. For instance, 2D nanomaterials composed by MoCoFeS supported on reduced graphene oxide (rGO) showed good electrochemical performance for HER [25]. Additionally, Figure 3 reveals that iron-doped tungsten oxide nanoplate supported on rGO nanocomposite (FeWOxP/rGO) exhibited excellent electrocatalytic activity towards HER due to the coupled synergic effect between many oxygen vacancies formation on tungsten oxide in the nanoplate structure of Fe-WOxP and rGO nanosheet [26]. Moreover, monodisperse PdRuNi nanoparticles decorated on graphene oxide were synthesized showing an exceptional performance and stability [27].

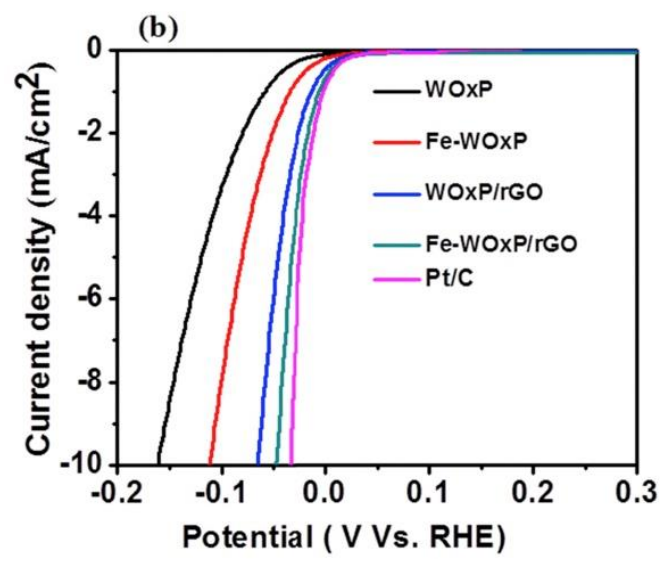

Figure 3. Hydrogen evolution on WOxP, Fe-WOxP, WOx/PrGO, Fe-WOxP/rGO, and $\mathrm{Pt} / \mathrm{C}$ recorded in $0.5 \mathrm{M} \mathrm{H}_{2} \mathrm{SO}_{4}$ at $2 \mathrm{mV} \mathrm{s}^{-1}$. Reproduced with permission (ref. 26).

Furthermore, a reduced graphene oxide/metallic $\mathrm{MoSe} 2: \mathrm{Cu}$ nanosheet has been synthesized and shown to be effective, as the $\mathrm{Cu}$ doping and the interface effect between MoSe2 and rGO increased the conductivity of the material, resulting in an active and stable catalyst [28]. 
Finally, Table 1 reports and compares the main physicochemical properties and catalytic performances towards the HER of non-precious metal graphene catalysts.

Table 1. Physicochemical properties of non-precious metal graphene catalysts.

\begin{tabular}{|c|c|c|c|c|c|}
\hline Catalyst & $\begin{array}{l}\text { Non metal } \\
\text { Doping } \\
\text { Elements }\end{array}$ & $\begin{array}{c}\text { Metal } \\
\text { doping } \\
\text { elements }\end{array}$ & $\begin{array}{c}\text { Onset } \\
\text { overpotential } \\
(\mathrm{mV} \text { vs } \mathrm{RHE})\end{array}$ & $\begin{array}{l}\text { Tafel slope } \\
\left(\mathrm{mV} \cdot \mathrm{dec}^{-1}\right)\end{array}$ & Ref. \\
\hline $\begin{array}{l}\text { Ultrathin graphene } \\
\text { shells encapsulated } \\
\text { in a uniform CoNi } \\
\text { nanoalloy }\end{array}$ & - & $\mathrm{Co}, \mathrm{Ni}$ & 142 & 107 & [12] \\
\hline $\begin{array}{l}\text { Plasma-etching on } \\
\text { S-graphene }\end{array}$ & $S$ & - & 178 & 86 & [19] \\
\hline $\begin{array}{l}\text { NS-doped } \\
\text { nanoporous } \\
\text { graphene (NS-500) }\end{array}$ & $\mathrm{N}, \mathrm{S}$ & - & 280 & 80.5 & {$[6]$} \\
\hline $\begin{array}{l}\text { Nitrogen and } \\
\text { phosphorus dual- } \\
\text { doped multilayer } \\
\text { graphene }\end{array}$ & $\mathrm{N}, \mathrm{P}$ & - & 120 & 79 & {$[16]$} \\
\hline $\begin{array}{l}\text { MoP nanoparticle } \\
\text { supported on N, P- } \\
\text { codoped reduced } \\
\text { graphene oxides }\end{array}$ & $\mathrm{N}, \mathrm{P}$ & Mo & 115 & 54 & [13] \\
\hline $\begin{array}{l}\text { B-substituted } \\
\text { graphene }\end{array}$ & - & B & $\sim 200$ & $\sim 99$ & [10] \\
\hline Ni-doped graphene & - & $\mathrm{Ni}$ & 50 & 45 & [15] \\
\hline $\begin{array}{l}\text { Ultrasmall } \\
\text { ruthenium } \\
\text { phosphide }\end{array}$ & - & $\mathrm{Ru}$ & 13 & 56 & [17] \\
\hline
\end{tabular}




\begin{tabular}{|c|c|c|c|c|c|}
\hline $\begin{array}{l}\text { nanoparticles } \\
\text { grown on reduced } \\
\text { graphene oxide } \\
\text { nanosheets }\end{array}$ & & & & & \\
\hline $\begin{array}{l}\text { Co, N-codoped } \\
\text { carbon nanotube } \\
(\mathrm{CNT}) \text { /graphene } \\
\text { heterostructure } \\
\text { bifunctional } \\
\text { catalyst }\end{array}$ & $\mathrm{N}$ & $\mathrm{Co}$ & 123 & 67 & [18] \\
\hline $\begin{array}{l}\mathrm{Cu}, \text { Co-embedded } \\
\text { nitrogen-enriched } \\
\text { mesoporous carbon } \\
\text { framework }\end{array}$ & $\mathrm{N}$ & $\mathrm{Cu}, \mathrm{Co}$ & 145 & 80 & [20] \\
\hline $\begin{array}{l}\text { MoCoFeS/reduced } \\
\text { graphene oxide }\end{array}$ & $\mathrm{S}$ & $\mathrm{Mo}, \mathrm{Co}, \mathrm{Fe}$ & 110 & 50 & [22] \\
\hline $\begin{array}{l}\text { Phosphine reduced } \\
\text { an iron-doped } \\
\text { tungsten oxide } \\
\text { nanoplate/reduced } \\
\text { graphene oxide } \\
\text { nanocomposite }\end{array}$ & $\mathrm{P}$ & $\overline{\mathrm{Fe}, \mathrm{W}}$ & 55 & 42 & {$[25]$} \\
\hline
\end{tabular}

\section{Summary}

Herein the general concepts of electrolysers are discussed, with special attention to graphene-based electrocatalysts. Electrolyser is a crucial technology for the desired hydrogen economy since it has the potential to provide pure hydrogen to fuel cells. With the aim to solve the principal catalytic problems at the cathode electrode of this device, the new advances in non-precious metal graphene materials is provided and a summary of the main catalytic properties are reported in Table 1. Along the manuscript, it was 
described diverse methodologies to modify graphene, and therefore its physicochemical properties, with the aim of tailor the properties for future design and synthesis of innovative and sustainable catalysts towards HER. Thus, the current work may help to improve the fabrication of novel electrodes in order to decrease the cost and to enhance the performance of electrolysers.

\section{Acknowledgments}

The Spanish Ministry of Economy and Competitiveness (MINECO) has supported this work under project ENE2017-83976-C2-2-R (co-funded by FEDER). G.G. acknowledges the Viera y Clavijo program (ACIISI \& ULL) for financial support.

\section{References}

[1] S. Solomon, G. Plattner, R. Knutti, P. Friedlingstein, Irreversible climate change due to carbon dioxide emissions, (2009).

[2] A.J. Bard, L.R. Faulkner, Electrochemical Methods: Fundamentals and Applications, 2nd Editio, JOHN WILEY \& SONS, INC., New York, 2001.

[3] M.M. Mench, Fuel Cell Engines, 2008. doi:10.1002/9780470209769.

[4] J.A. Turner, J.A. Turner, Sustainable Hydrogen Production, 972 (2014). doi:10.1126/science.1103197.

[5] M. Rashid, M.K. Al Mesfer, H. Naseem, M. Danish, Hydrogen Production by Water Electrolysis : A Review of Alkaline Water Electrolysis, PEM Water Electrolysis and High Temperature Water Electrolysis, (2015) 80-93.

[6] N. Sammes, Fuel Cell Technology: Reaching Towards Commercialization In Engineering Materials and Processes Series, Springer-V, London, UK, 2006.

[7] Y. Ito, W. Cong, T. Fujita, Z. Tang, M. Chen, High Catalytic Activity of Nitrogen and Sulfur Co-Doped Nanoporous Graphene in the Hydrogen Evolution Reaction ** Angewandte, (2014) 1-7. doi:10.1002/anie.201410050.

[8] H. Wang, T. Maiyalagan, X. Wang, Review on Recent Progress in NitrogenDoped Graphene: Synthesis, Characterization, and Its Potential Applications, ACS Catal. 2 (2012) 781-794. doi:10.1021/cs200652y.

[9] X. Huang, X. Qi, F. Boey, H. Zhang, Graphene-based composites, Chem. Soc. Rev. 41 (2012) 666-686. doi:10.1039/C1CS15078B.

[10] L.M.L.M. Rivera, S. Fajardo, M.C.M. del C. Arévalo, G. García, E. Pastor, S- 
and N-Doped Graphene Nanomaterials for the Oxygen Reduction Reaction, Catalysts. 7 (2017) 278. doi:10.3390/catal7090278.

[11] B.R. Sathe, X. Zou, T. Asefa, Catalysis Science \& Technology electrocatalytic activity for hydrogen evolution reaction $\dagger$, (2014) 2023-2030. doi:10.1039/c4cy00075g.

[12] L.D. Carr, M.T. Lusk, Graphene gets designer defects, Nat. Publ. Gr. 5 (2010) 316-317. doi:10.1038/nnano.2010.93.

[13] H. Jiang, Y. Zhu, Y. Su, Y. Yao, Y. Liu, X. Yang, C. Li, graphene : e ffi cient metal-free electrocatalysts for the hydrogen evolution reaction $\dagger$, J. Mater. Chem. A Mater. Energy Sustain. 3 (2015) 12642-12645. doi:10.1039/C5TA02792F.

[14] J. Zhang, X. Wang, Y. Xue, Z. Xu, J. Pei, Z. Zhuang, Self-Assembly PrecursorDerived MoP Supported on N , P-Codoped Reduced Graphene Oxides as E ffi cient Catalysts for Hydrogen Evolution Reaction, Inorg. Chem. 57 (2018) 1385913865. doi:10.1021/acs.inorgchem.8b02359.

[15] S. Agnoli, M. Favaro, Doping graphene with boron : a review of synthesis, J. Mater. Chem. A Mater. Energy Sustain. 4 (2016) 5002-5025. doi:10.1039/C5TA10599D.

[16] Y. Tian, Z. Wei, X. Wang, S. Peng, X. Zhang, ScienceDirect Plasma-etched, Sdoped graphene for effective hydrogen evolution reaction, 2 (2016) 2-10. doi:10.1016/j.ijhydene.2016.09.142.

[17] H. Qiu, Y. Ito, W. Cong, Y. Tan, P. Liu, A. Hirata, T. Fujita, Z. Tang, M. Chen, Nanoporous Graphene with Single-Atom Nickel Dopants : An Efficient and Stable Catalyst for Electrochemical Hydrogen Production Angewandte, (2015) 14031-14035. doi:10.1002/anie.201507381.

[18] T. Liu, S. Wang, Q. Zhang, L. Chen, W. Hu, C.M. Li, Ultrasmall Ru 2 P nanoparticles on graphene : a electrocatalyst in both acidic and alkaline media $\uparrow$, (2018) 3343-3346. doi:10.1039/c8cc01166d.

[19] J. Deng, P. Ren, D. Deng, X. Bao, Enhanced electron penetration through an ultrathin graphene layer for highly efficient catalysis of the hydrogen evolution reaction, Angew. Chemie - Int. Ed. 54 (2015) 2100-2104. doi:10.1002/anie.201409524.

[20] D.C. Liu Yang, Yanlong Lv, Co, N-codoped nanotube/graphene 1D/2D heterostructure for efficient oxygen reduction and hydrogen evolution reactions, J. Mater. Chem. A. (2018). doi:10.1039/C7TA11140A. 
[21] M. Kuang, Q. Wang, P. Han, G. Zheng, Cu , Co-Embedded N-Enriched Mesoporous Carbon for Efficient Oxygen Reduction and Hydrogen Evolution Reactions, 1700193 (2017) 1-8. doi:10.1002/aenm.201700193.

[22] Y. Qu, Y. Ke, Y. Shao, W. Chen, C.T. Kwok, X. Shi, H. Pan, E ff ect of Curvature on the Hydrogen Evolution Reaction of Graphene, (2018). doi:10.1021/acs.jpcc.8b06750.

[23] J. Su, Y. Yang, G. Xia, J. Chen, P. Jiang, Q. Chen, Ruthenium-cobalt nanoalloys encapsulated in nitrogen-doped graphene as active electrocatalysts for producing hydrogen in alkaline media, Nat. Commun. 8 (2017) 14969. doi:10.1038/ncomms14969.

[24] J. Wang, G. Wang, S. Miao, J. Li, X. Bao, Graphene-supported iron-based nanoparticles encapsulated in nitrogen-doped carbon as a synergistic catalyst for hydrogen evolution and oxygen reduction reactions, Faraday Discuss. 176 (2014) 135-151. doi:10.1039/C4FD00123K.

[25] M. Bagher, P. Salarizadeh, S. Mohammad, MoCoFeS hybridized with reduced graphene oxide as a new electrocatalyst for hydrogen evolution reaction, 711 (2018) 32-36. doi:10.1016/j.cplett.2018.09.025.

[26] T.H. Wondimu, G. Chen, D.M. Kabtamu, H. Chen, A.W. Bayeh, H. Huang, C.H. Wang, ScienceDirect Highly efficient and durable phosphine reduced iron-doped tungsten oxide / reduced graphene oxide nanocomposites for the hydrogen evolution reaction, Int. J. Hydrogen Energy. 43 (2018) 6481-6490. doi:10.1016/j.ijhydene.2018.02.080.

[27] S. Communication, B. Sen, B. Demirkan, S.K. Gu, F. Sen, ScienceDirect Trimetallic PdRuNi nanocomposites decorated on graphene oxide : A superior catalyst for the hydrogen evolution reaction, 3 (2018) 1-9. doi:10.1016/j.ijhydene.2018.07.122.

[28] H.-Y. He, Z. He, Q. Shen, Reduced graphene oxide/metallic MoSe2: Cu nanosheet nanostructures grown by a chemical process for highly efficient water splitting, Mater. Res. Bull. 111 (2019) 183-190. doi:https://doi.org/10.1016/j.materresbull.2018.11.009. 